\title{
Lagrangian Coherent Structure Analysis of Terminal Winds: Three-Dimensionality, Intramodel Variations, and Flight Analyses
}

\author{
Brent Knutson, ${ }^{1}$ Wenbo Tang, ${ }^{1}$ and Pak Wai Chan ${ }^{2}$ \\ ${ }^{1}$ School of Mathematical and Statistical Sciences, Arizona State University, Tempe, AZ 85287, USA \\ ${ }^{2}$ Hong Kong Observatory, Hong Kong \\ Correspondence should be addressed to Wenbo Tang; wenbo.tang@asu.edu
}

Received 2 November 2014; Revised 20 February 2015; Accepted 20 February 2015

Academic Editor: Luis Gimeno

Copyright ( $\odot 2015$ Brent Knutson et al. This is an open access article distributed under the Creative Commons Attribution License, which permits unrestricted use, distribution, and reproduction in any medium, provided the original work is properly cited.

We present a study of three-dimensional Lagrangian coherent structures (LCS) near the Hong Kong International Airport and relate to previous developments of two-dimensional (2D) LCS analyses. The LCS are contrasted among three independent models and against 2D coherent Doppler light detection and ranging (LIDAR) data. Addition of the velocity information perpendicular to the LIDAR scanning cone helps solidify flow structures inferred from previous studies; contrast among models reveals the intramodel variability; and comparison with flight data evaluates the performance among models in terms of Lagrangian analyses. We find that, while the three models and the LIDAR do recover similar features of the windshear experienced by a landing aircraft (along the landing trajectory), their Lagrangian signatures over the entire domain are quite different-a portion of each numerical model captures certain features resembling those LCS extracted from independent 2D LIDAR analyses based on observations.

\section{Introduction}

A Lagrangian framework for terminal wind hazard detection near Hong Kong International Airport (HKIA) has been recently developed and locations of such disturbances have been compared against flight data [1-3]. The approach outlined in this series of work focuses on obtaining signatures of convergence and divergence of fluid parcel trajectories based on two-dimensional (2D), near-ground velocity data retrieved from light detection and ranging (LIDAR) equipment [4]. The disturbances extracted from the Lagrangian methods are found to be in close proximity of real jolts experienced by landing aircraft. Validating with lengthy flight data over several months, it is found that this approach outperforms traditional Eulerian measures, such as velocity fluctuation measurements [5] as they provide better Receiver Operating Characteristic (ROC) graphs [6, 7], and matches closely with an operational algorithm based on a scanning pattern that follows the actual aircraft landing trajectories [8].

One limitation of the aforementioned methodology is the lack of three-dimensional (3D) data from the 2D LIDAR output. Indeed this is a common limitation shared by all other methods based on 2D LIDAR data, and the Lagrangian framework outperforms traditional methods in part due to its capability to better infer the signatures transversal to the 2D plane-position-indicator (PPI) scanning cone. Variational wind retrieval algorithms in three dimensions are also available [9-11], but they are more time-consuming and relevant to operational forecasts at HKIA, and PPI scans are only available at a few elevation angles. Henceforth, it is beneficial to verify that sophisticated results based on $2 \mathrm{D}$ data are useful in operational applications.

In this study, we aim to explore to what extent the transversal signatures inferred from $2 \mathrm{D}$ scans represent true 3D structures-Do we find correspondence of 3D vertical structures at the locations where $2 \mathrm{D}$ convergence and divergence are the strongest, at least near the center of the LIDAR scanning cone? Does this interpretation successfully extrapolate to data at the peripheral of the LIDAR scanning cone, where the vertical elevation could be over 100 meters above mean sea level, and an argument of strong twodimensionality near ground may not apply? What extra information does $3 \mathrm{D}$ data reveal that is absent from $2 \mathrm{D}$ analyses? 
Towards this end, we have generated three independent numerical simulations of the regional atmospheric flows near HKIA, for a case of strong windshear on December $27 \mathrm{th}, 2009$. This case corresponds to an airstream associated with a ridge of high pressure along the southeastern coast of China meeting a cold front from inland, resulting in aircraft diverting to Shenzhen because they could not land at HKIA [12]. Two of the simulations are based on numerical weather prediction models-the Regional Atmospheric Modelling System (RAMS) [13] and the Weather Research and Forecasting model (WRF) [14]; the third simulation uses the FLOWSTAR package, which is analytically based and depends more on the terrain data than the physics [15]. In terms of the initialization of the simulations, the two weather forecast models are driven by global forecast system (GFS) data [16] and use nested grids to achieve high resolution over HKIA, whereas the latter uses upstream observational data (independent from LIDAR) as the constant boundary conditions for computation of a steady state solution.

To realize our goal on validation of the $2 \mathrm{D}$ analyses, we make the following comparisons. Firstly, we contrast LCS among different models to obtain a full picture of the $3 \mathrm{D}$ flow structures. This comparison reveals the variability among models. Secondly, within each model, we compare LCS obtained from full 3D data to those derived from $2 \mathrm{D}$ wind fields interpolated on the LIDAR scanning cone. Two schemes are considered for the $2 \mathrm{D}$ data generation. One is the horizontal wind speed interpolated directly from 3D model. This wind field closely mimics the resolved model flow, so the comparison directly reflects $2 \mathrm{D}$ signatures of a $3 \mathrm{D}$ field. The other uses line-of-sight (LOS) velocity from the models and goes through the $2 \mathrm{D}$ wind retrieval scheme [4]. The retrieved wind is then used to generate 2D LCS. This effectively mimics the procedure of LIDAR measurements-LCS generation. Consequently 2D information loss and modeling assumed in the wind retrieval scheme are tested with 3D data. Thirdly, we contrast the 3D and 2D LCS from models with 2D LCS obtained from the actual LIDAR measurements to check for any correspondence. Lastly, we compare the LCS from these analyses with data collected from a landing aircraft. This brings all models and schemes to the ultimate test for possible operational implementation.

The rest of the paper is organized as follows. In Section 2 we briefly review the wind retrieval, extrapolation, and LCS generation algorithms. In Section 3 we introduce the three numerical model data sets. In Section 4 we discuss the various comparisons among $3 \mathrm{D}, 2 \mathrm{D}$, and measurement data. In Section 5, we draw conclusions and discuss further studies underway.

\section{Wind Retrieval and LCS Generation Algorithms}

We briefly summarize the algorithms used to generate $2 \mathrm{D}$ wind retrieval from LIDAR scans and to extract LCS based on the retrieved data. The $2 \mathrm{D}$ wind retrieval algorithm for
LIDAR is modified from a two-step variational method for RADAR [11]. The cost function $J$ to be minimized is given by

$$
\begin{aligned}
J(u, v)=J_{1}+ & J_{2}+J_{3}+J_{4}+J_{5}+J_{6} \\
=\sum_{i, j}\{ & W_{1}\left[\left(u-u_{B}\right)^{2}+\left(v-v_{B}\right)^{2}\right] \\
& +W_{2}\left(v_{r}-v_{r}^{\mathrm{obs}}\right)+W_{3}(\Delta x)^{2}\left(\frac{\partial u}{\partial x}+\frac{\partial v}{\partial y}\right)^{2} \\
& +W_{4}(\Delta x)^{2}\left(\frac{\partial v}{\partial x}-\frac{\partial u}{\partial y}\right)^{2} \\
& +W_{5}(\Delta x)^{4}\left(\nabla^{2} u+\nabla^{2} v\right)^{2} \\
& \left.+\sum_{n}\left[W_{6}\left(\frac{\partial v_{r}^{\mathrm{obs}}}{\partial t}+u \frac{\partial v_{r}^{\mathrm{obs}}}{\partial x}+v \frac{\partial v_{r}^{\mathrm{obs}}}{\partial y}\right)^{2}\right]\right\},
\end{aligned}
$$

where $u$ and $v$ are the components of the retrieved wind field, subscript $B$ is the background field, generated from LIDAR radial velocity in the way described in [11], $v_{r}$ is the retrieved radial velocity, superscript obs is the observed values, $i$ and $j$ are the horizontal grid point, and $n$ is the time index (three consecutive scans are used in each analysis). The weights are $W_{1}=0.1$ (after the first step retrieval), $W_{2}=1, W_{3}=W_{4}=$ $W_{5}=0.1$, and $W_{6}=10^{4}$. They are chosen empirically in this paper to ensure that the constraints have proper orders of magnitude. The model-emulated LOS velocity is subject to this retrieval algorithm for emulated $2 \mathrm{D}$ wind and subsequent LCS analyses. For more discussions of this algorithm, the readers are referred to $[2,4]$.

The retrieved wind is extrapolated beyond the LIDAR resolved range as a global linear flow that best fits the $2 \mathrm{D}$ wind retrieval [1]. This best reveals the nonlinearity inside the LIDAR observational domain while it avoids addition of extra nonlinearity from data outside when they are completely unknown. In terms of the current study, this extrapolation is applied to the true and emulated LIDAR data (so as to measure the performance of the 2D LCS retrieval algorithm). The numerical models have data coverage outside of the LIDAR range and those data are used in the $3 \mathrm{D}$ computations as they provide the true LCS pertinent to the corresponding velocity fields.

For a Cartesian grid $\mathscr{G}$ in a rectangular region, where the coordinate axes have been chosen such that the domain center corresponds to the origin, the closest linear incompressible flow which minimizes error in the Euclidean norm is

$$
\mathbf{v}_{L}(\mathbf{x}, t)=\left(\begin{array}{cc}
\frac{\left\langle x u_{1}-y u_{2}\right\rangle}{\left\langle x^{2}+y^{2}\right\rangle} & \frac{\left\langle y u_{1}\right\rangle}{\left\langle y^{2}\right\rangle} \\
\frac{\left\langle x u_{2}\right\rangle}{\left\langle x^{2}\right\rangle} & \frac{\left\langle y u_{2}-x u_{1}\right\rangle}{\left\langle x^{2}+y^{2}\right\rangle}
\end{array}\right) \mathbf{x}+\left(\begin{array}{c}
\left\langle u_{1}\right\rangle \\
\left\langle u_{2}\right\rangle
\end{array}\right)
$$


where $\langle g\rangle=\sum_{i=1}^{m} \sum_{j=1}^{n} g\left(x_{i}, y_{j}\right) /(m \times n)$ is the spatial average of a function $g$ over the grid $\mathscr{G}$. letting

The global flow is constructed on the whole plane by

$$
\mathbf{v}=\mathbf{v}_{L}+\left(\mathbf{u}-\mathbf{v}_{L}\right) f,
$$

where $f$ is a filter function that takes value 1 inside a subset $\mathscr{G}^{0}$ of $\mathscr{G}$ and value 0 in the exterior of $\mathscr{G}$. In between we have a buffer zone of width $\Delta$ where $f$ smoothly transition between 1 and 0 . This allows smooth trajectories to leave the LIDAR domain.

We use a common measure, the finite-time Lyapunov exponents (FTLE), to reveal the LCS field. Given a velocity field (2D or $3 \mathrm{D})$, the Lagrangian position of a fluid parcel $\mathbf{x}\left(t ; \mathbf{x}_{0}, t_{0}\right)$ at time $t$ which started at location $\mathbf{x}_{0}$ and time $t_{0}$ can be computed numerically, and the FTLE is defined as

$$
\begin{aligned}
& \sigma\left(t ; \mathbf{x}_{0}, t_{0}\right) \\
& \quad=\frac{1}{2\left|t-t_{0}\right|} \ln \left\{\lambda_{\max }\left(\left[\frac{\partial \mathbf{x}\left(t ; \mathbf{x}_{0}, t_{0}\right)}{\partial \mathbf{x}_{0}}\right]^{T} \frac{\partial \mathbf{x}\left(t ; \mathbf{x}_{0}, t_{0}\right)}{\partial \mathbf{x}_{0}}\right)\right\} .
\end{aligned}
$$

An auxiliary grid approach is used to approximate the FTLE field with better accuracy [17]. Also, trajectories near ground are allowed to slide along the surface with horizontal velocity at $10 \mathrm{~m}$. This helps remove structures due to the noslip boundary conditions and better reveals the structures more relevant to airflow disturbances that affect the airport.

The FTLE field [3] and its associated gradients [6] have been used to compare LCS signatures of airflow hazard and jolts experienced from flight data. We will contrast the LCS analyzed here with flight data for the corresponding event in a similar fashion in Section 4.

\section{Numerical Model Data for the Windshear Event}

The numerical model data based on RAMS and FLOWSTAR have been reported previously $[12,18]$, but they focus on comparisons between the headwind profile from measurements onboard a flight and in numerical predictions. Both models capture the general trend of the measured headwind profile. In this study, these two model data sets, along with a third one generated using WRF, are used for LCS analyses.

The RAMS model is nested within the $20 \mathrm{~km}$ resolution Operational Regional Spectral Model (ORSM) of the Hong Kong Observatory (HKO). RAMS was run with nested grids having spatial resolutions of $4 \mathrm{~km}, 800 \mathrm{~m}$, and $200 \mathrm{~m}$ with two-way nesting. The smallest nest is sufficient to resolve the mountains on Lantau Island, immediately south of HKIA. The Mellor-Yamada turbulence parameterization scheme was used in the first grid and the Deardorff scheme in the other two grids. The model run started at 1200 UTC, 26 December 2009, and was carried out for $12 \mathrm{~h}$.

The FLOWSTAR model is based on topographic data and meteorological inputs and generates steady-state solutions of velocity. The boundary conditions for the FLOWSTAR runs were defined using the observed $10 \mathrm{~m}$ wind upstream of Lantau Island and by the radiosonde ascent for midnight UTC 27 December 2009 at King's park for the wind speed and direction, vertical temperature structure, and hence buoyancy frequency. Three cases were considered for the wind speed and direction and boundary layer height. In this study we use the data set that best matches the measured headwind profile. This corresponds to an upstream wind speed of $7.3 \mathrm{~ms}^{-1}$ at $z=10 \mathrm{~m}$ height, wind direction of $140^{\circ}$, boundary layer height $h_{0}=400 \mathrm{~m}$, buoyancy frequency profile $N=0 \mathrm{~s}^{-1}$ for heights $z<h_{0}, N=0.0124 \mathrm{~s}^{-1}$ for heights $z>h_{0}$, temperature step of $7.19^{\circ} \mathrm{C}$ at $z=h_{0}$, and surface roughness of $0.5 \mathrm{~m}$. The readers are referred to $[12,18]$ for more thorough discussions of these two models.

Finally, the WRF model is initiated from the GFS data and runs with nested grids having spatial resolutions of $51.2 \mathrm{~km}$, $12.8 \mathrm{~km}, 3.2 \mathrm{~km}, 800 \mathrm{~m}$, and $200 \mathrm{~m}$ with two-way nesting. The model is centered at $22.313 \mathrm{~N}$ and $113.92 \mathrm{E}$. In an attempt to match the high horizontal resolution, each nest also had 85 vertical levels. In the 3 coarsest grids the YSU (Yonsei University scheme) boundary layer parameterization was used, whereas in the 2 finest resolution nests the boundary layer parameterization was turned off in order to have full 3D diffusion. Full diffusion (diff_opt $=2$ ) was selected, paired with the 1.5 order TKE prediction for turbulence parameterization. Each nest used the Dudhia shortwave radiation and RRTM longwave radiation schemes. Five-layer thermal diffusion was used as the land surface option, whereas the MM5 similarity scheme was selected for the surface layer and the Kessler scheme was used for microphysics. No cumulus parameterization was used. The simulation is initiated at 1800 UTC, 26 December 2009, and ran for $6 \mathrm{~h}$.

As a first comparison, we contrast some Eulerian velocity data among models and measurements in Figure 1, for a case of missed approach. The approach is from left to right, as indicated by the black arrow above Figure 1(a). Figures $1(a)-1(c)$ show comparison of the headwind for the three numerical models along the landing corridor. The aircraft trajectory is also shown in each of the panel as the thick black solid curve. It is seen that all three models capture a region of strong headwind near the runway threshold. Flying through this patch leads to significant windshear. The two regional models appear to capture the same trend of this patch from bottom left to top right. This patch is more columnar in the FLOWSTAR data. The sharp transition in the FLOWSTAR model at $400 \mathrm{~m}$ indicates the height of the inversion layer. To better infer the flow topology, we use the velocity vectors projected on the vertical plane of the landing corridor (headwind and vertical velocity). It seems that RAMS has the weakest vertical velocity in this plane; FLOWSTAR shows more wave undulation as the aircraft approaches the runway threshold, yet WRF shows a hint of flow reversal at $-1 \mathrm{NM}$ from the runway threshold between 300 and $500 \mathrm{~m}$ in altitude. This feature possibly leads to strong LCS. In Figure 1(d), the headwind profile is shown from different data sets. In this panel, the thick black solid curve is the actual measurement of headwind onboard the landing aircraft. The magenta curve shows the headwind profile 


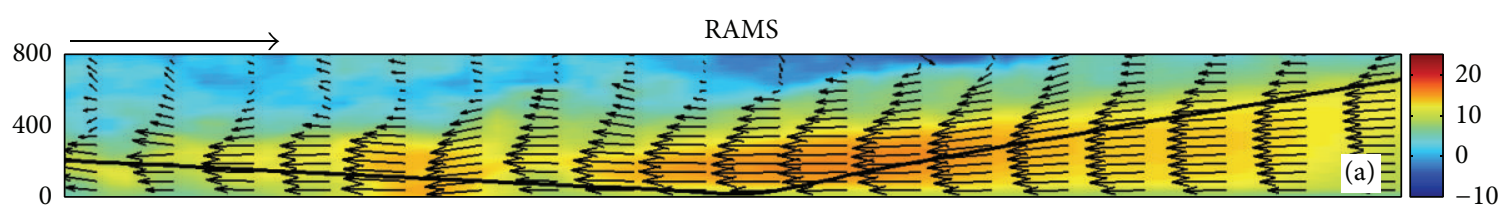

FLOWSTAR

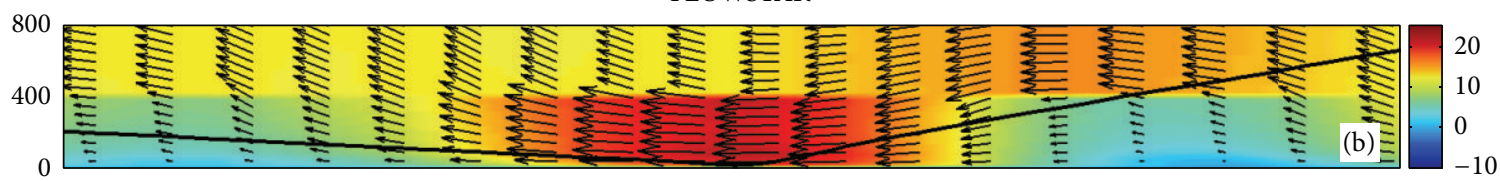

WRF
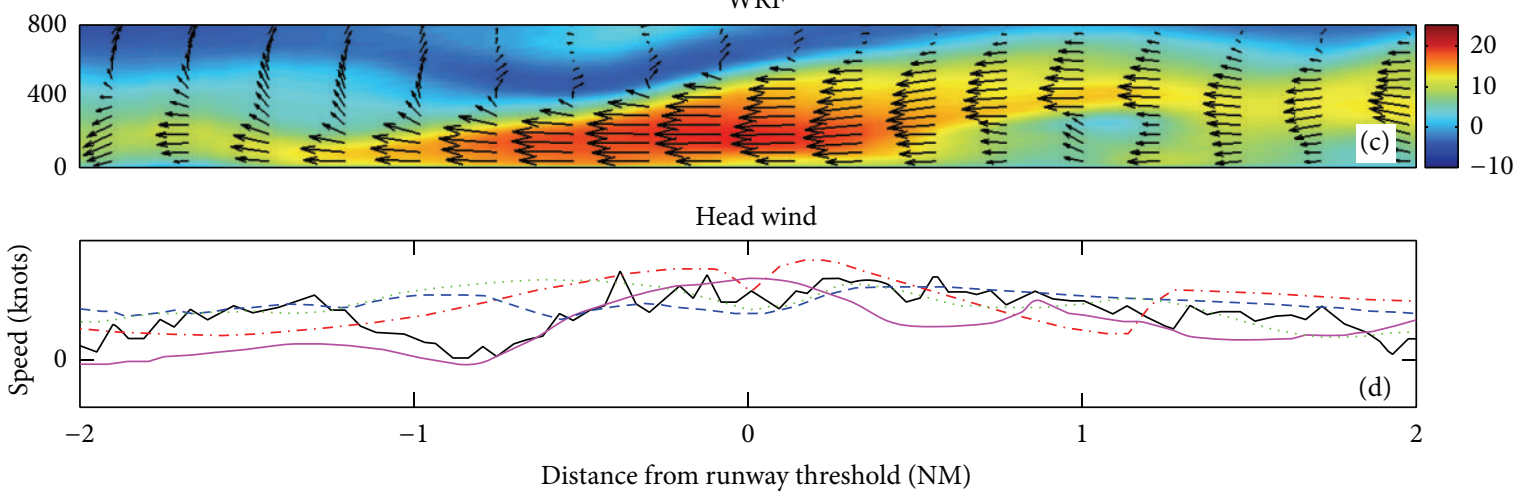

FIGURE 1: (a)-(c) Horizontal wind speeds (in knots) for RAMS, FLOWSTAR, and WRF, respectively, along the landing corridor. The thick black solid curve in each figure is the landing trajectory. The vector field is the plot of headwind and vertical velocity along the landing corridor, where hints of coherent structures can be seen. (d) Headwind comparison between measurements and models. Thick black solid curve: onboard measurement. Magenta curve: LIDAR measurements along the PPI scanning cone. Blue dashed curve: interpolated headwind from RAMS. Red dash-dotted curve: interpolated headwind from FLOWSTAR. Green dotted curve: interpolated headwind from WRF. The black arrow above (a) indicates direction of flight.

extracted from LIDAR conical scans. This data closely follows the velocity ramp from onboard measurement at about -1 nautical mile (NM) from the runway threshold. The blue dashed curve (RAMS), red dash-dotted curve (FLOWSTAR), and green dotted curve (WRF) differ from the onboard measurement further, but they do capture the general trend of the windshear (with weaker velocity gradients). A cross correlation study shows that, at the respective maxima, the LIDAR data is $71 \%$ correlated with onboard head wind measurements, followed by WRF (63\%), FLOWSTAR (62\%), and RAMS (56\%). Lagrangian signatures of these resolved flow data are discussed in the following section.

\section{LCS Comparisons}

Before comparison with 2D data sets, we first analyze the 3D LCS structure from various models and their implications on the actual terrain-induced turbulence patches.

4.1. Three-Dimensional Features of LCS. The three-dimensional features of the LCS for the three data sets are shown in Figure 2. These features are highlighted with two vertical slices in the domain. One vertical slice is along the southern runway, highlighting structures the airplane experiences when landing. The other vertical slice is perpendicular to the southern runway, so features transversal to the runway can be seen. The airplane landing trajectory is shown in each of the panels as the thick black curve. Direction of flight is again marked by a red arrow in Figure 2(a), along the vertical slice aligned with the southern runway. Relevant position of this trajectory with the coherent structures, the Lantau Island, and the airport are easily seen. The red dots at sea surface height in each panel mark the distances for each nautical mile from the runway threshold, which is located at the lowest point of the plane trajectory. To aid the interpretation of the coherent structures, selected fluid parcel trajectories (thin black curves with black arrows) near ground around the airport are also shown to reveal the actual atmospheric motion. These trajectories are generated from the timedependent velocity data in each of the flow models and they interact with the airplane near the runway threshold.

The left column of Figure 2 shows FTLE obtained from RAMS. The center column shows those from FLOWSTAR and the right column shows those from WRF. The top panels correspond to the forward time FTLE highlighting separation and the bottom panels are those from backward time FTLE highlighting attraction. The integration time for these FTLE fields is 6 minutes. This is sufficient to capture shortterm flow structures that will be averaged out during longterm integrations, yet important for flight safety. It is also consistent with the integration time from $2 \mathrm{D}$ velocity data, so the features among these data sets can be directly compared. 


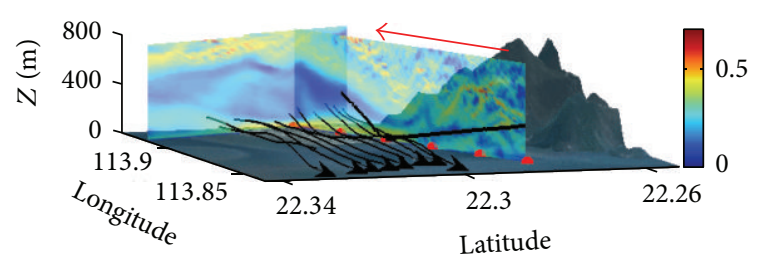

(a)

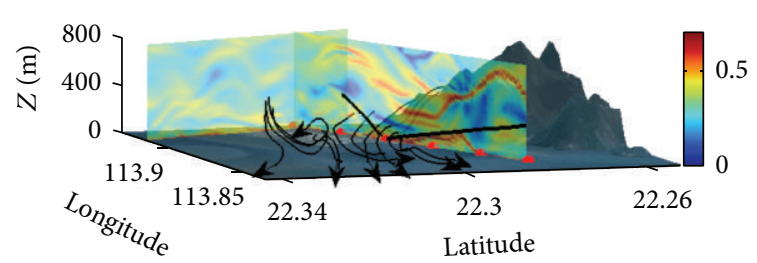

(c)

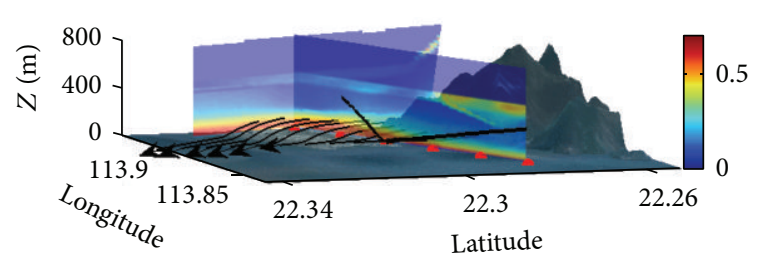

(e)

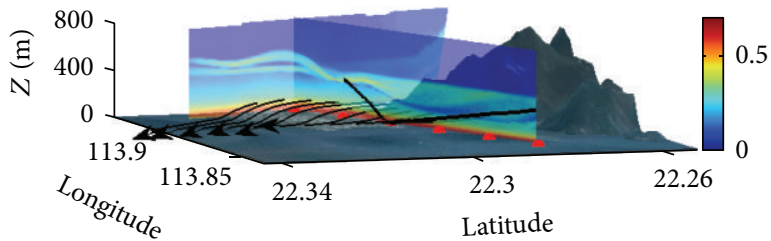

(b)

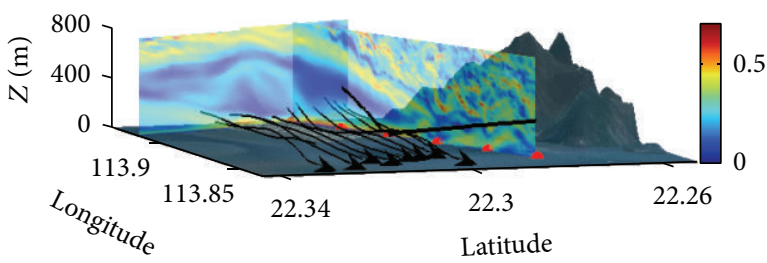

(d)

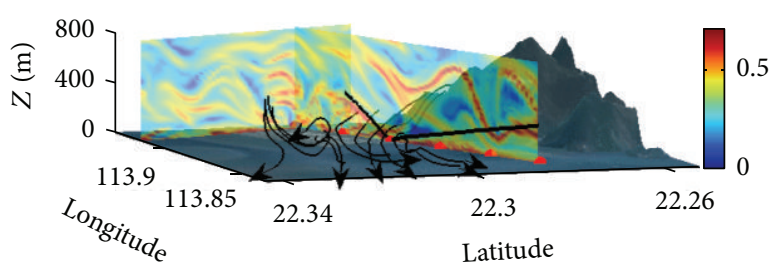

(f)

FIGURE 2: Lagrangian coherent structures from different data sets and their relative positions to the Lantau island. The opaque color maps are the vertical slices of the FTLE fields along and perpendicular to the runways, intersecting at the southern runway threshold towards the west. The thick black curve in each panel is the airplane landing trajectory. The thin black curves are fluid trajectories highlighting the structures, with black arrows indicating direction of flow. The panels are, from left to right, data from RAMS, FLOWSTAR, and WRF, respectively. The top panels are the forward time FTLE and the bottom panels are the backward time FTLE. Axes are given in (d). The red arrow indicates direction of flight. The red dots on sea surface level mark every nautical mile towards the runway threshold. The runway threshold is located at the lowest point of the flight trajectory.

Judging from the orientation of the structures, it can be seen that the two regional atmospheric models (RAMS and WRF) behave quite similarly, with wavy structures between 400 and $600 \mathrm{~m}$ altitude (cf. (a), (d), (c), and (f). The vertical slices are shown up to $800 \mathrm{~m}$ ). Some structures are also seen near the bottom around the airport, and between 200 and $400 \mathrm{~m}$ relatively less separation is seen. The FLOWSTAR data carries less structures ((b) and (e)) regarding the fact that major features are seen near the bottom and around 400-500 m, with low separation in between these heights. These distinguished structures in FLOWSTAR data can be explained due to strong shear across the inversion layer.

We closely inspect the fluid parcel trajectories to further interpret the structures. Consistent with the respective velocities, the RAMS ((a) and (d)) and WRF ((c) and (f)) data show easterlies/east-southeasterlies, whereas the FLOWSTAR data shows a southeasterly. In RAMS data, several streaks of waves along the runway can be identified; their nonlinear motion leads up to the corrugated patches seen near the bottom of the domain. The FLOWSTAR data shows little horizontal separation of trajectories, but some vertical motion can be identified. The WRF data is most interesting, as several vortex rolls aligned roughly in the east-west direction can be identified, some of which cross the runway threshold.
Overall, the two regional models show good correspondence between the data, with the exception that the vertical velocity in RAMS seems to be less than that of WRF. As comparison, the FLOWSTAR data show less detailed features of coherent structures, probably due to the heavily idealized assumptions.

4.2. Comparisons between Full and Conical LCS. We employ two schemes to contrast the LCS from fully $3 \mathrm{D}$ data and $2 \mathrm{D}$ emulated data. In the first scheme, 3D LCS are interpolated along the LIDAR scanning cone and compared to those generated from $2 \mathrm{D}$ velocity along the same cone. This scheme reveals whether it will be plausible to use $2 \mathrm{D}$ information to infer 3D structures. To further mimic the process of generating LCS from LIDAR line-of-sight (LOS) velocity, the second scheme emulates LOS velocity scans from simulation data and generates 2D wind retrieval and then finds the coherent structures. These results are also compared to actual LIDAR based FTLE. For easy handling of lack of data when the LIDAR scanning cone intersects with mountain topography, we use the FDFTLE algorithm [1] to extend the data and allow smooth integration of trajectories. This avoids artificial structures associated with trajectories running into the Lantau Mountain. In this subsection, the three model 
flows are considered, and the benchmarks and the 2D LCS are approximations to these benchmarks.

We show in Figure 3 the FTLE fields interpolated along the LIDAR scanning cone. This figure serves as the reference for comparisons with approximations. The panels are similar to Figure 2, with the left, center, and right columns corresponding to RAMS, FLOWSTAR, and WRF data sets, and the top and bottom rows correspond to the forward and backward time integrations. The integration time is 6 minutes. For easy comparison and feature identification we only focus on a region immediately west of the southern runway threshold (threshold marked by the black circle), corresponding to the smallest nest in RAMS simulation. The topography near the airport is shown as the black isocontours and the landing trajectory is shown as the thick black line. The southern LIDAR (square) and $-1 \mathrm{NM}$ before the runway threshold (diamond), where a strong windshear was reported, are also marked for comparison. As seen, the two regional models show similar features of flow structures, especially near the southern LIDAR, with some east-west ridge structure above the west end of the airport. This consistency could be due to both models being driven from global model data, and hence they bear similar features from the larger nests towards the smallest nests. Further away from the airport, the WRF data shows more coherent patterns indicating more organized convergence and divergence of flow, whereas the RAMS data shows more scattered features, indicating less organized separation. The stronger coherence in WRF data could be due to the stronger vertical velocity (cf. Figure 1 and trajectory comparisons in Figure 2) giving rise to better defined vortex rolls. The FLOWSTAR data is oriented slightly towards the north-west, consistent with the incoming flow direction. The arc pattern on the left end of this data set is due to the transition of structures from below to above the inversion layer, where strong shear gives rise to a highlighting layer of separation, as seen in Figures 2(b) and 2(e). Below this layer (inside the arc towards the LIDAR), some features of the forward time FTLE can still be identified (Figure 3(b)). In all models, the strong windshear at $-1 \mathrm{NM}$ can be associated with coherent structures highlighted as the FTLE ridge.

To better understand the role of highlighting FTLE at $-1 \mathrm{NM}$, we plot groups of black dots, indicating positions of fluid parcels every 2 minutes. These trajectories are initiated at the diamond, at $190 \mathrm{~m}$ elevation, where the windshear is reported. The initial height of the trajectories is determined based on the elevation of the PPI scanning cone at this location. The trajectories are integrated using the full 3D data. The deformation of these dots indicates the local nonlinear behaviors of the flow. As seen, in RAMS data in Figures 3(a) and $3(\mathrm{~d})$, there is trajectory separation transversal to the local structures in both forward and backward time, resulting in the FTLE ridges nearby. The forward time trajectories in FLOWSTAR data show very weak stretching associated with a low FTLE value ridge. The backward trajectories of FLOWSTAR mostly show contraction in the meridional direction and very weak separation in the zonal direction, hence giving no highlighting structure near the $-1 \mathrm{NM}$ location. The WRF trajectories show stretching along a local structure in forward time and attraction transversal to a local structure in backward time. As such, all FTLE behaviors are accounted for with the behaviors of the local trajectories.

In Figure 4, we show those 2D results using horizontal wind data on the PPI scanning cone. For easy comparison, the layout is precisely the same as those in Figure 3. The data sets are, from left to right, RAMS, FLOWSTAR, and WRF. Plots in the top row show forward time structures and plots in the bottom row show backward time structures. Trajectories near $-1 \mathrm{NM}$ from runway threshold based on the 2D interpolated data are again shown to explain the local FTLE behavior and compare to those from Figure 3. It can be seen that the shapes of local deformation of the trajectories are similar to those in Figure 3.

To better quantify the match between Figures 3 and 4, we use a local correlation map. Because flow structures relevant to flight operations are relatively small, we compare features of size $1 \mathrm{~km} \times 1 \mathrm{~km}$. The correlation between the 3D interpolated and 2D FTLE fields of each patch of $1 \mathrm{~km}^{2}$ is computed at each patch center and plotted in Figure 5. The layout is the same as the previous two figures for the three data sets and two directions of integration. In regions of red color, there is match between features, whereas in regions of blue color, there are mismatches. This leads to the question of what kind of three-dimensionality leads to the discrepancies in these two plots. Before explaining such discrepancies, we first locate regions where strong similarities can be found. The thick black line segments in Figures 3 and 4 correspond to structures where correspondence can be found between 3D and 2D extraction. Such correspondence is mainly seen in RAMS and WRF data, near the LIDAR (marked by the square). This is can be explained by the local two-dimensionality near ground, around the center of the LIDAR scanning cone. At $-1 \mathrm{NM}$ and beyond, the LIDAR cone is already at $190 \mathrm{~m}$. Structures may vary strongly below the cone, resulting in the mismatch in the interpretation of the FTLE ridges. The FLOWSTAR data has the least correspondence between the $3 \mathrm{D}$ and $2 \mathrm{D}$ analyses. There is a weak ridge in the $2 \mathrm{D}$ computation that could be related to the more pronounced ridge in $3 \mathrm{D}$ analyses. We use the thin dashed line to identify this possible match.

In order to understand the discrepancies in the $3 \mathrm{D}$ and $2 \mathrm{D}$ analyses, we focus on a few regions where the mismatch is pronounced. These regions are highlighted by the black ellipses in Figures 3 and 4, with centers marked by the black dots (note their correspondence in the blue regions in Figure 5). We are not concerned with the big red spot near LIDAR because it is due to vertical shear near ground arising from the no-slip boundary condition (even though we allowed trajectories to continue to flow at the speed of $10 \mathrm{~m}$ height, the horizontal velocity is still significantly lower than those in higher elevations). For RAMS data, the two analyses are quite similar, and hence we choose an FTLE structure that is absent in the $3 \mathrm{D}$ analyses yet present in $2 \mathrm{D}$ analyses. This region is only about $1 \mathrm{NM}$ northwest of the LIDAR; hence being able to interpret this structure helps us understand possible conflicts near $100 \mathrm{~m}$ elevation. For FLOWSTAR data, we choose a region immediately north of the -1 NM location. As indicated above, the match in forward time structures is with questions and the backward time structure does not 

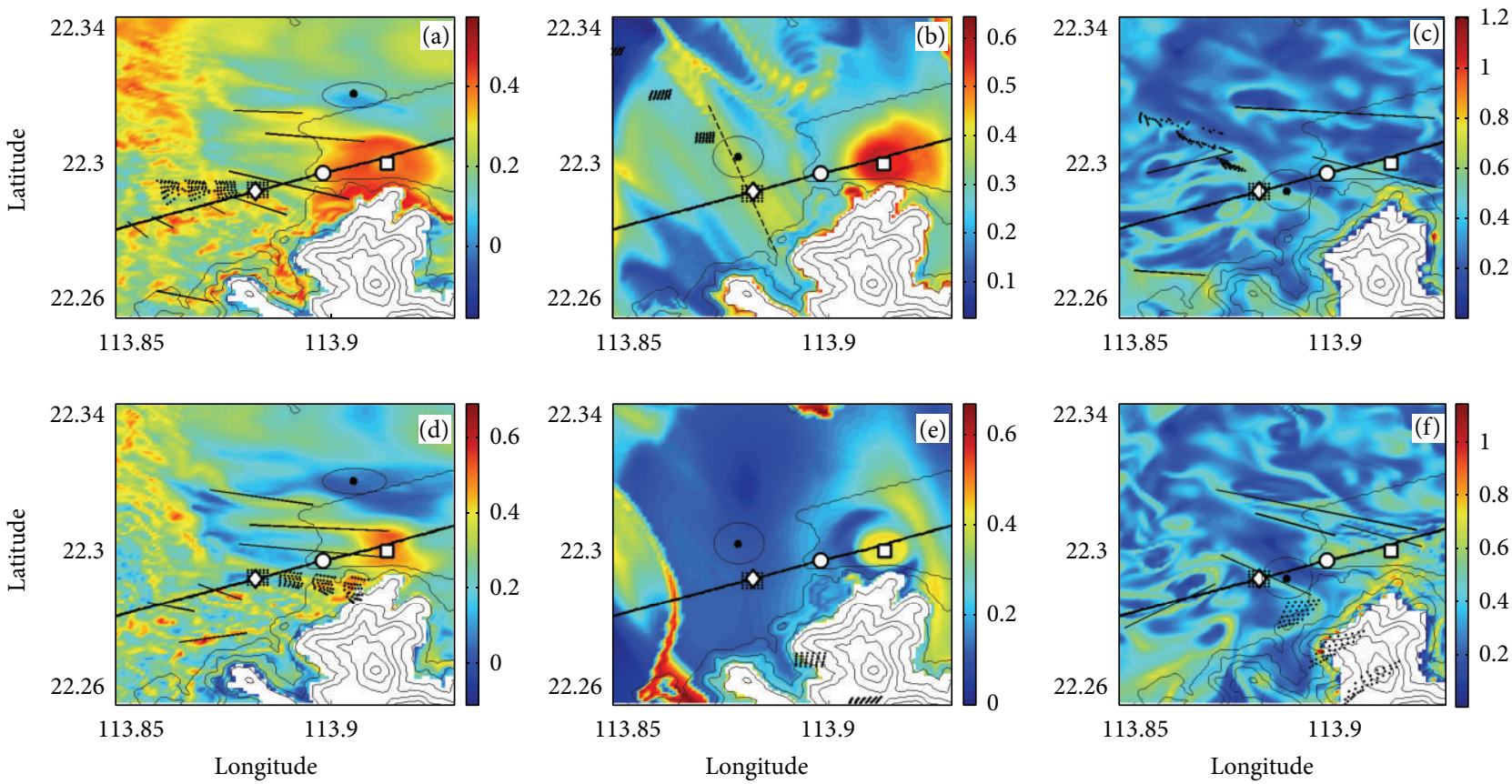

FIGURE 3: FTLE fields interpolated on the LIDAR scanning cone. The panels are, from left to right, data from RAMS, FLOWSTAR, and WRF, respectively. The top panels are the forward time FTLE and the bottom panels are the backward time FTLE. The black straight lines mark locations of great similarity in FTLE with Figure 4, and the black circles mark locations of a few mismatches with Figure 4.
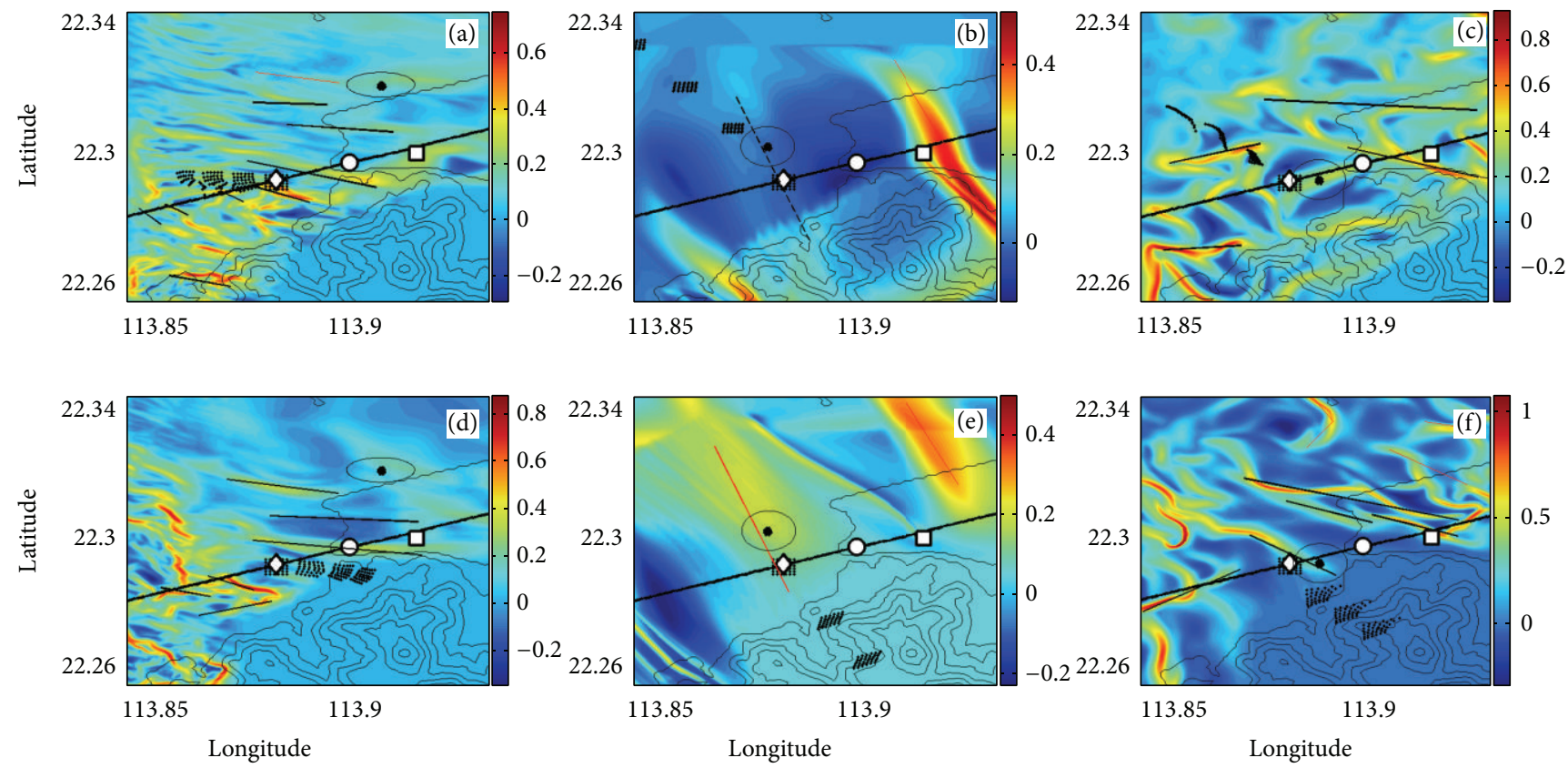

FIGURE 4: FTLE fields based on 2D emulated data on the LIDAR scanning cone. The panels are, from left to right, data from RAMS, FLOWSTAR, and WRF, respectively. The top panels are the forward time FTLE and the bottom panels are the backward time FTLE. The black straight lines and the black circles mark the same locations as seen in Figure 3 for reference.

show much correspondence at this location. For WRF data, to the east of the $-1 \mathrm{NM}$ location, the $3 \mathrm{D}$ analyses show a structure transversal to the landing trajectory yet $2 \mathrm{D}$ analyses show a structure aligned with the landing trajectory. We focus on this region to see what trajectory behaviors lead to these results.
We explain the difference in the plots in Figure 6, for the three data sets. In all three panels, trajectories based on 3D data are shown in black, and trajectories based on 2D data are shown in red. The directions of the flow are indicated by the text arrows. In Figure 6(a), there is probably only weak horizontal separation in the $3 \mathrm{D}$ trajectories (note that the FTLE 

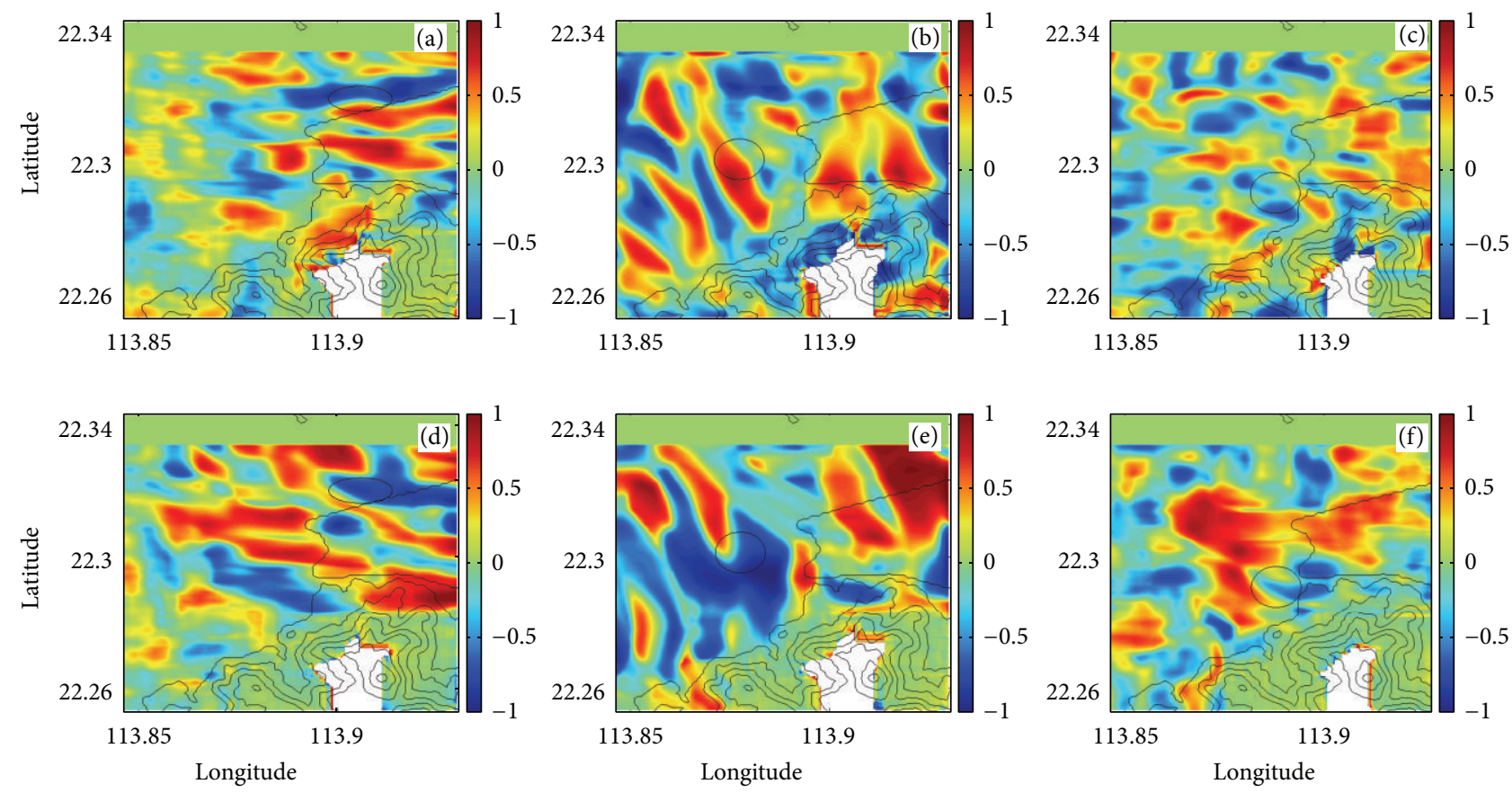

Figure 5: Localized correlation map between Figures 3 and 4 . The sizes of the local regions are $1 \mathrm{~km} \times 1 \mathrm{~km}$ patches. The black circles mark the mismatches discussed in Figures 3 and 4.

value is not 0 at this location in Figure 3(a)). The trajectories just follow a wave undulation across the airport in the eastwest direction. Because 2D trajectories leave the domain and use linear extrapolation, it gives rise to a bit larger stretching and a local FTLE ridge. From Figure 6(b), it is seen that trajectories in FLOWSTAR data also bear very weak horizontal separation. The ridge structure in Figure 3(b) is mainly due to such a weak separation. The horizontal discrepancies in the backward time trajectories are better seen in Figures 3(e) and $4(\mathrm{e})$. Both show a bit of separation in the visible domain. As the trajectories leave the domain, the $3 \mathrm{D}$ trajectories follow an external shear flow that has no separation (given as the upstream condition), yet the $2 \mathrm{D}$ trajectories again undergo a linear extrapolation, where trajectories continue to separate, giving rise to the slightly larger FTLE value seen in Figure 4(e). The most interesting comparison is in Figure 6(c) for WRF data. Here, a large vertical displacement is seen in forward time trajectories. In the 3D trajectories, towards the northeast of the examined initial conditions, the trajectories go underneath those starting towards the southwest. Those starting towards the southwest pick up elevation quickly and form large separation both vertically and horizontally. In fact these trajectories are caught in a local vortex roll. The LIDAR scan sees first horizontal contraction (as the east and west trajectories flip over) at low elevation near the LIDAR and then horizontal separation in the higher elevation (as the west trajectories rotate with the vortex roll). As seen, the incorporation of the vertical stretching contributes to significant change of structure at this particular location.

In the context of flight operations, horizontal windshear is of more concern as it directly relates to the lift of the airplane. the vertical separation could be due to nonparallel shear leading nearby vertical trajectories into vastly different positions. The airplane is less sensitive to this kind of separation. As such, we compute a partial FTLE field, based on the separation of nearby horizontal trajectories (which are advanced with the full $3 \mathrm{D}$ velocity). This measure excludes the effects of direct vertical separation but does incorporate horizontal separation as the trajectories enter different elevations. Its use in atmospheric flows has been justified in [19]. The results of this computation are shown in Figure 7. Figure 7 has the same layout as Figures 3 and 4 . A most notable difference between Figures 7 and 3 is the red spots near LIDAR. They disappear in Figure 7 because the vertical shear near ground has been excluded as the FTLE computation only considered horizontal separations. As seen, these figures bear much better correspondence with the $2 \mathrm{D}$ analyses, especially for RAMS and FLOWSTAR data (when the vertical velocity was smaller as compared to WRF). Correspondence in WRF between Figures 4 and 7 is still better than that between Figures 3 and 4 .

We show in Figure 8 comparison among LCS from LOS velocities. The panels are, from left to right, RAMS, FLOWSTAR, WRF, and LIDAR scan. The plots in the top row show forward time LCS and those in the bottom row show backward time LCS. For the model data, we first compute the LOS velocity on the LIDAR scanning cone. Based on this data, we process $2 \mathrm{D}$ variational retrieval outlined in Section 2. The retrieved velocity is then processed with the FDFTLE algorithm. This fully emulates the cycle of realistic LIDAR retrieval that is operational at HKIA. We first contrast the LOS based 2D LCS to the previous $3 \mathrm{D}$ and $2 \mathrm{D}$ LCS 


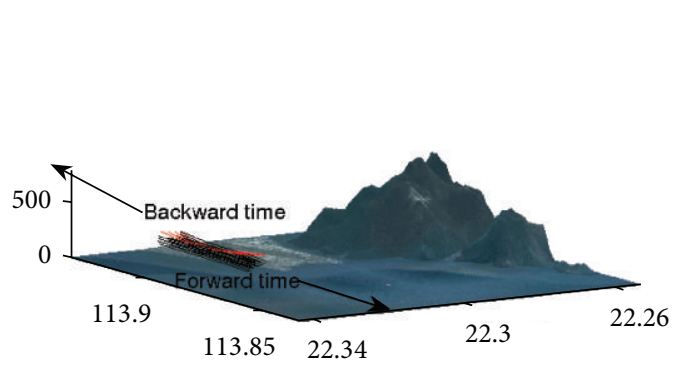

(a)

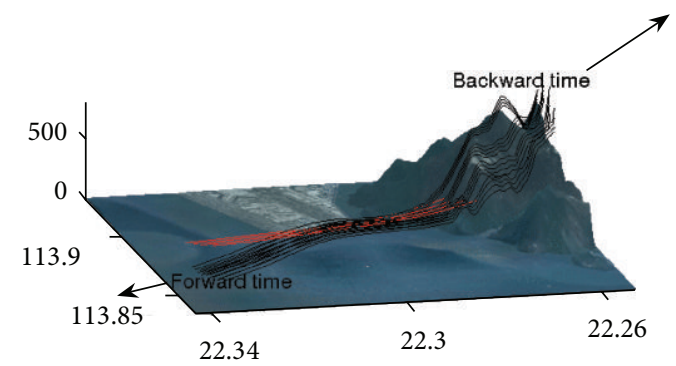

(b)

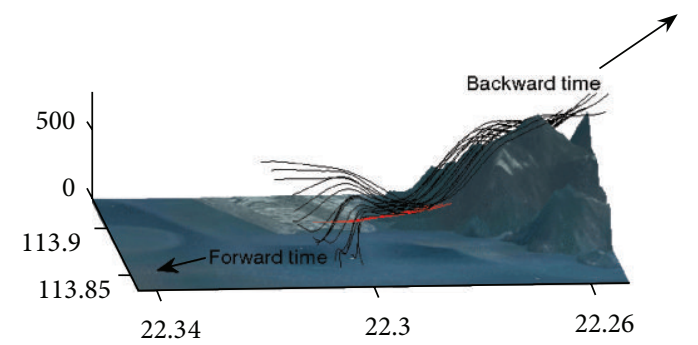

(c)

FIGURE 6: Trajectory comparison between 3D data and 2D data on the LIDAR cone. The panels are, from left to right, data from RAMS, FLOWSTAR, and WRF, respectively.
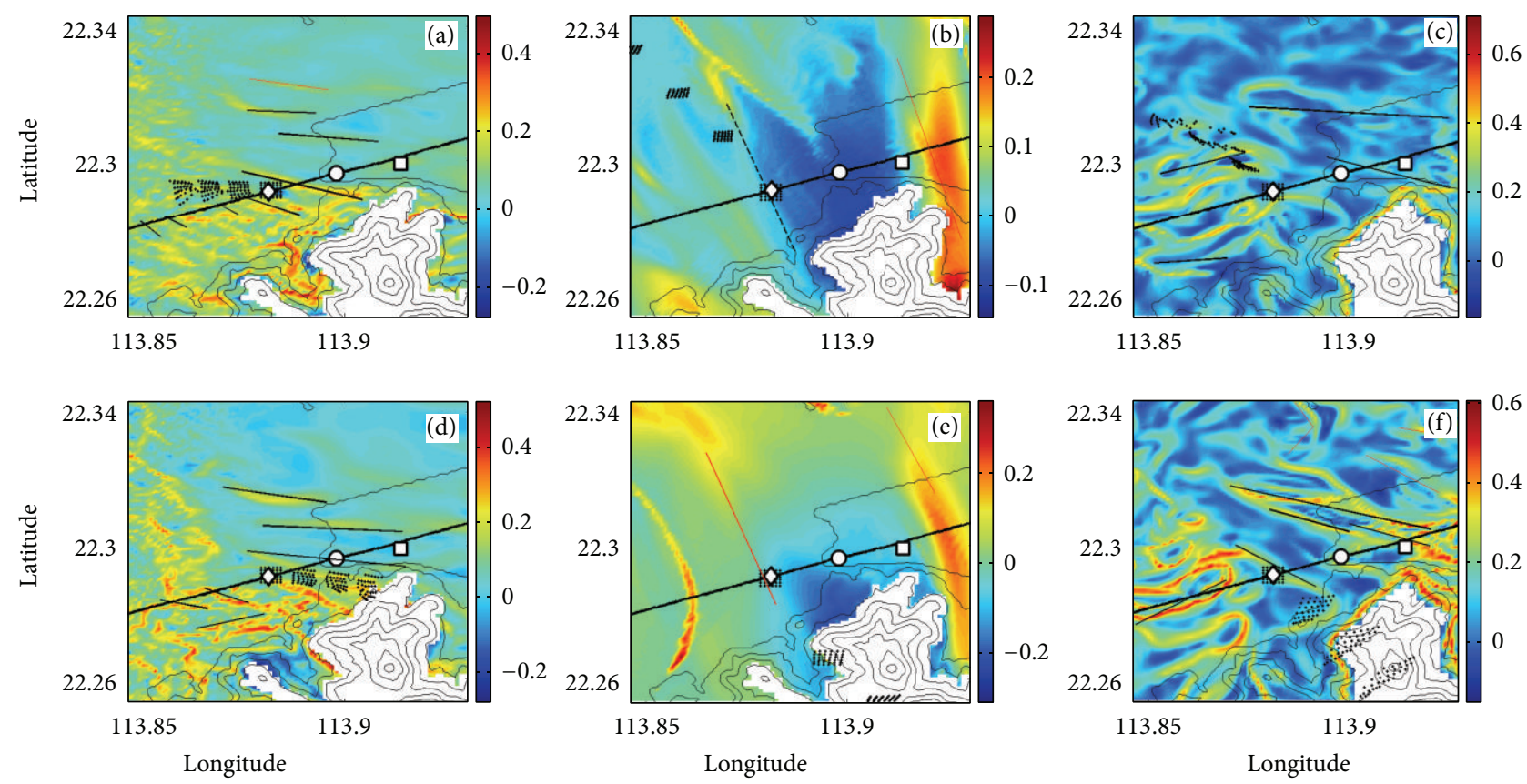

FIGURE 7: FTLE fields computed from just horizontal stretching. The panels are, from left to right, data from RAMS, FLOWSTAR, and WRF, respectively. The top panels are the forward time FTLE and the bottom panels are the backward time FTLE.

results. It is quite clear that all plots compare well with features seen in Figure 4 in the respective model data sets. There is further degradation from the $3 \mathrm{D}$ results, but the structures near LIDAR seem to be robust even undergoing the many approximations. Those structures between the $-1 \mathrm{NM}$ windshear location and LIDAR seem to preserve their shape especially well.
As a comparison to real data, we also show, in Figures 8(d) and $8(\mathrm{~h})$, LCS extracted from LIDAR observed LOS. The WRF data seem to have the best match with LIDAR generated LCS, although FLOWSTAR shows better correspondence in the orientation of the structures. In addition, the RAMS data also has good correspondence with LIDAR data around the LIDAR. It is especially interesting to note that the mismatch 

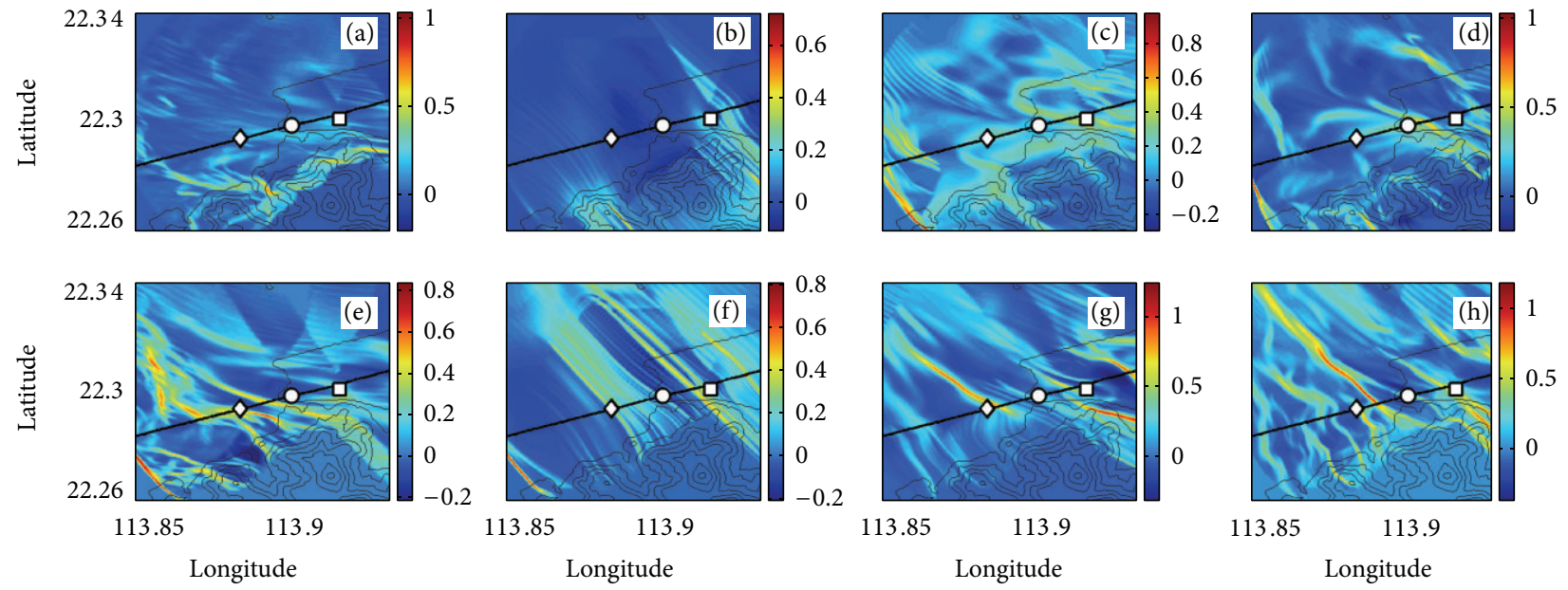

FIGURE 8: FTLE fields computed from LOS velocity and 2D variational retrieval. The panels are, from left to right, data from RAMS, FLOWSTAR, WRF, and LIDAR, respectively. The top panels are the forward time FTLE and the bottom panels are the backward time FTLE.

structure aligned with the landing trajectory in Figure 4(c) is also present in Figures 8(c) and 8(d), further ascertaining the match between the WRF data set and the benchmark.

Using cross correlation among two-dimensional data sets $[20,21]$ in Figure 8, we confirm that the max correlation between LIDAR and WRF is 35\% for forward FTLE and 33\% for backward FTLE, between LIDAR and FLOWSTAR is 13\% for forward FTLE and 32\% for backward FTLE and between LIDAR and RAMS is $30 \%$ for forward FTLE and $20 \%$ for backward FTLE. This is generally consistent with the visual observations. Note that we only allow the shift in both longitudinal and latitudinal directions to be $\pm 1 \mathrm{~km}$ to ensure that the correlation is meaningfully through nearby structures.

4.3. Comparisons with Onboard Measurements. In [6], it is shown that the projected FTLE gradient, defined as

$$
\sigma^{P}=|\nabla \sigma \cdot \mathbf{e}|,
$$

where $\mathbf{e}$ is the direction along the glide path, yields better Receiver Operating Characteristic (ROC) graphs as compared to the FTLE value or norm of FTLE. As such, in this subsection we contrast FTLE value $\sigma$ and the projected FTLE gradient $\sigma^{P}$ for results from the various schemes at hand. In order to provide a ROC graph, large numbers of cases need to be analyzed. Here we only focus on one reported windshear case and contrast only the backward time measures, which are more relevant to jolts experienced by the aircraft.

We show our detailed comparison in Figure 9. The left three columns of Figure 9 are from three-dimensional simulation data (RAMS, FLOWSTAR, and WRF, resp.) and the last column is from LIDAR scans. The $x$-axis is the distance from the runway threshold along the glide path, and the $y$-axis is the relevant Lagrangian measures. The top panels are from the FTLE values $\sigma$ and the bottom panels are from the projected FTLE gradients $\sigma^{P}$. The gradients are also scaled by $10 \mathrm{NM}$ to place the curves in similar scale. In each panel, the black curve is the vertical acceleration measured onboard. As seen, there are several spikes from
$-1 \mathrm{NM}$ towards the runway threshold, where a very large spike appears. Windshear was reported at $-1 \mathrm{NM}$. In the left three columns, the blue, red, and green curves correspond to $3 \mathrm{D}$ (reduced FTLE in Figure 7), 2D, and LOS based FTLE interpolations. In the right column, since only 2D information is available, the blue curves are LOS based FTLE. These curves are shifted up by 0.4 for clarity of the figure.

The RAMS data in Figures 9(a) and 9(e) show consistency with the major spikes. In particular, the $3 \mathrm{D}$ (blue) and $2 \mathrm{D}$ (red) FTLE from RAMS do have (relatively small) spike at $-1 \mathrm{NM}$. Although a spike is not found precisely at $-1 \mathrm{NM}$ for the LOS based FTLE (green) in Figure 9(a), a major peak is found nearby. As such, in the projected gradient field $\sigma^{P}$ (Figure 9(e)), a spike is found at $-1 \mathrm{NM}$ for the green curve. In fact, in this plot, several other spikes are identified leading up to the spike at the runway threshold, with good correspondence in the precise locations of spikes from all three schemes of FTLE extraction. As for the major peak at the runway threshold, minor spikes can be found to the right of this peak in all schemes.

The 3D (blue) and 2D (red) FLOWSTAR data in Figures $9(\mathrm{~b})$ and $9(\mathrm{f})$ show little correspondence with the actual vertical acceleration experienced onboard. Again, this may be due to the fact that FLOWSTAR is more driven by the topography instead of physics, and so a precise match cannot be found. However, we still do identify spikes in the LOS based $\sigma$ and $\sigma^{P}$ fields (green) that can be related to the major spikes at $-1 \mathrm{NM}$ and at the runway threshold.

The WRF data in Figures 9(c) and 9(g) picks up the spikes at -1 NM most significantly. Both the 2D (red) and LOS based (green) $\sigma$ and $\sigma^{P}$ show spikes at $-1 \mathrm{NM}$, yet the 3D (blue) and LOS based (green) $\sigma$ and $\sigma^{P}$ show spikes at the runway threshold. A minor peak can also be identified in the $3 \mathrm{D}$ (blue) curves around $-1 \mathrm{NM}$. This minor structure can be identified near the diamond in Figure 7(f).

Most relevant to operations, the LIDAR scan shows strong spiking behavior at $-1 \mathrm{NM}$ and the runway threshold, indicating the good correspondence between the two 


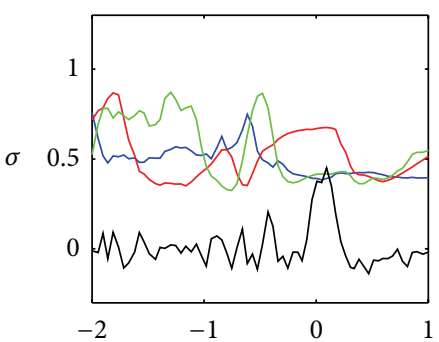

(a)

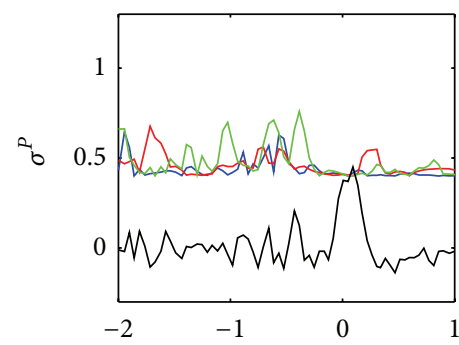

(e)

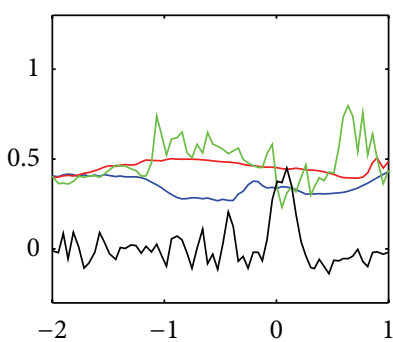

(b)

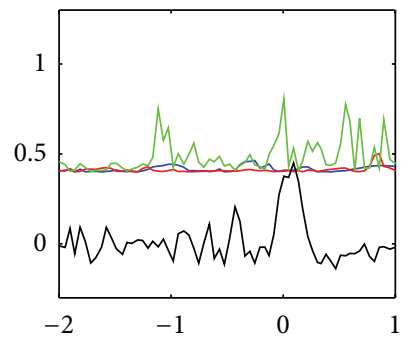

(f)

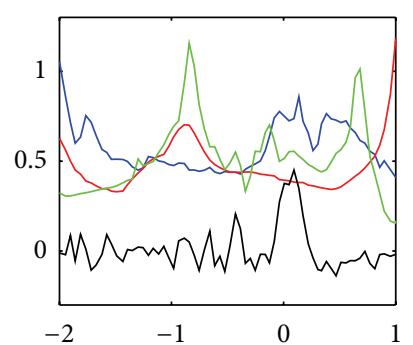

(c)

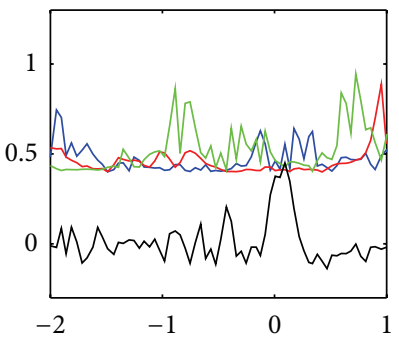

(g)

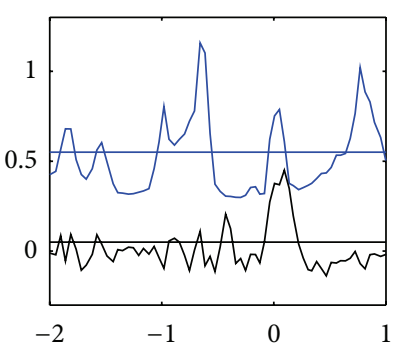

(d)

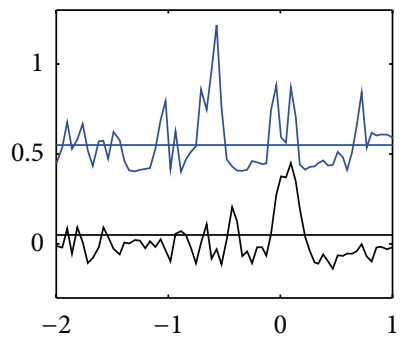

(h)

FIGURE 9: Lagrangian measures compared to vertical acceleration measured onboard an aircraft. From left to right, the data is from RAMS, FLOWSTAR, WRF, and LIDAR scans. Only backward time measures are compared since they are relevant to jolts experienced onboard. The top panels are the FTLE values interpolated along the glide path, and the bottom panels are the projected FTLE gradient. A strong windshear is reported at $-1 \mathrm{NM}$ from the runway threshold ( 0 in the $x$-axis). The black curves in each panel are vertical acceleration measured from the aircraft. For the left three columns, the blue, red, and green curves correspond to data interpolated from the reduced FTLE, 2D FTLE, and LOS based FTLE. In the right column (from actual LIDAR), the blue curves are LOS based FTLE.

measurement data. Comparing the LOS based FTLE, it can be seen that WRF data most closely mimic those from the LIDAR in Figure 9.

To quantify the correlation among the many graphs shown, we focus on the relation between the vertical acceleration profile and the LIDAR based FTLE (the rightmost column in Figure 9), as well as the relation between the LOS based FTLE from the three models (green curves in the left three columns of Figure 9) and the LIDAR based FTLE. The first comparison shows the effectiveness of the LIDAR based algorithm for real-time forecasting, whereas the second shows the performance of the models to observations. Note that since FTLE and vertical acceleration are two entirely different measures, one would not expect that the shape matches closely. As such, we first use the conditional probability developed in [3] to quantify the effectiveness of LIDAR based FTLE in detecting actual jolts.

Consider vertical acceleration greater than $0.05 \mathrm{~g}$, where $g$ is the gravitational acceleration, being strong jolts (spikes above the black horizontal line), we look for strong FTLE ridge $\left(>0.15 \mathrm{~s}^{-1}\right)$ in the vicinity $(150 \mathrm{~m})$ of the jolts (spikes above the blue horizontal line). The justification of these parameters is given in [3]. For the landing approach analyzed here, we find that 1 out of 7 jolt spikes is left unaccounted for, and 1 out of 6 FTLE ridges does not correspond to a nearby jolt. Using FTLE gradient, again, 1 out of 7 jolt spikes is left unaccounted for, and 3 out of 10 FTLE ridges do not correspond to a nearby jolt. In fact, using cross correlation, the max correlation between the FTLE field and the vertical acceleration profile is 55\%, whereas that between the FTLE gradient and the vertical acceleration is $57 \%$.

We use cross correlation to measure the correlation of the model data with LIDAR. At the respective maxima, in terms of the FTLE field, the LIDAR is $24 \%$ correlated to RAMS, $39 \%$ correlated to the FLOWSTAR, and 58\% correlated to the WRF. In terms of gradient, the LIDAR is $23 \%$ correlated with RAMS, 19\% correlated with FLOWSTAR, and 33\% correlated with WRF. The high correlation between the LOS based WRF data and LIDAR as well as the high correlation in their head wind profile led us to believe that WRF best captures the details of the flow variations close to observations.

\section{Conclusions}

The recently developed Lagrangian based turbulent structure extraction methods near the Hong Kong International Airport bring forward great promise in accurate detection of airflow hazards and prompt issuance of windshear warnings. The original method is based on two-dimensional scans from light detection and ranging equipment, going through a variational retrieval step. This huge loss of information necessitates the need to validate the $2 \mathrm{D}$ methodology against $3 \mathrm{D}$ data sets to (i) verify that the $2 \mathrm{D}$ interpretations of $3 \mathrm{D}$ structure are correct; (ii) test if the Lagrangian extraction methodology does capture major flow hazards; (iii) confirm that the LIDAR based data (which has already gone through averaging in the measurements and modeling in the variational retrieval step) is useful in representing the real atmosphere; and (iv) 
compare with actual flight data and verify the applicability of the entire Lagrangian based methodology.

In this study, we have conducted thorough comparisons to address the aforementioned needs. Our first comparison between full 3D LCS and 2D LCS from velocity projections onto the LIDAR scanning cone indicates that there could be mismatches when the stretching rate is evaluated from the vertical separations. A reduced FTLE based on just the horizontal separation of trajectories shows good correspondence between the $3 \mathrm{D}$ and $2 \mathrm{D}$ data, at least within 2 nautical miles from the LIDAR. This confirms that our interpretation of 3D structures from 2D signatures is valid, at least close to the LIDAR where the elevation is low.

Secondly, we emulate the LCS generation process as if the $3 \mathrm{D}$ velocity data is the atmospheric truth. Line-of-sight (LOS) velocity on the LIDAR scanning cone is interpolated and used to generate $2 \mathrm{D}$ velocity via a variational retrieval technique developed at HKIA. Our results show that, inside 2-nautical-mile radius from LIDAR, the LOS based LCS is again comparable to the reduced FTLE. This confirms the applicability using LIDAR based LCS to interpret true atmospheric structures.

Thirdly, in order to test if the model data are truthful to real atmospheric conditions, we compare the model based LIDAR emulated LCS to true LIDAR based LCS. It is found that each model captures some feature of the true LIDAR based LCS, yet those results produced from our WRF simulation match the best with LIDAR results. The close match between WRF and truth, as well as the close relation between 3D LCS and LOS based LCS from the WRF data set, suggests that the LIDAR based LCS can be used to infer structures in the real atmosphere.

Finally, we compare the LCS results and actual jolts experienced onboard an aircraft which attempted to land at the time of our analyses. It is found that our Lagrangian indicators (most pronouncedly from WRF and LIDAR) capture well the large accelerations experienced by the aircraft. This helps establish the point of using Lagrangian measures as operational tools in the detection of safety-threatening and comfort-compromising jolts during landings.

The close match between WRF and LIDAR data brings us hope to further refine our Lagrangian methodology by improving the forecasting model data. Currently we are working on using data assimilation to incorporate LIDAR scans into high resolution WRF model. Such results will be reported elsewhere.

\section{Conflict of Interests}

The authors declare that there is no conflict of interests regarding the publication of this paper.

\section{Acknowledgments}

The authors acknowledge Julian Hunt, David Carruthers, and Andrew Ellis for helpful discussions, for their assistance with the FLOWSTAR data. Wenbo Tang and Brent Knutson also acknowledge the support by the NSF Grant (ATM-0934592).

\section{References}

[1] W. Tang, P. W. Chan, and G. Haller, "Accurate extraction of Lagrangian coherent structures over finite domains with application to flight data analysis over Hong Kong international airport," Chaos, vol. 20, no. 1, Article ID 017502, 2010.

[2] W. Tang, P. W. Chan, and G. Haller, "Lagrangian coherent structure analysis of terminal winds detected by lidar. Part I. Turbulence structures," Journal of Applied Meteorology and Climatology, vol. 50, no. 2, pp. 325-338, 2011.

[3] W. Tang, P. W. Chan, and G. Haller, "Lagrangian coherent structure analysis of terminal winds detected by lidar. Part II: structure evolution and comparison with flight data," Journal of Applied Meteorology and Climatology, vol. 50, no. 10, pp. 21672183, 2011.

[4] P. W. Chan and A. M. Shao, "Depiction of complex airflow near Hong Kong International Airport using a Doppler LIDAR with a two-dimensional wind retrieval technique," Meteorologische Zeitschrift, vol. 16, no. 5, pp. 491-504, 2007.

[5] P. W. Chan and Y. F. Lee, "Application of short-range lidar in wind shear alerting," Journal of Atmospheric and Oceanic Technology, vol. 29, no. 2, pp. 207-220, 2012.

[6] H. A. Kafiabad, P. W. Chan, and G. Haller, "Lagrangian detection of wind shear for landing aircraft," Journal of Atmospheric and Oceanic Technology, vol. 30, no. 12, pp. 2808-2819, 2013.

[7] T. Fawcett, "An introduction to ROC analysis," Pattern Recognition Letters, vol. 27, no. 8, pp. 861-874, 2006.

[8] P. W. Chan, C. M. Shun, and K. C. Wu, "Operational lidar-based system for automatic windshear alerting at the Hong Kong international airport," in Proceedings of the 12th Conference on Aviation, Range, \& Aerospace, American Meteorological Society, Atlanta, Ga, USA, 2006.

[9] J. Sun, D. Flicker, and D. Lilly, "Recovery of three dimensional wind and temperature fields from simulated Doppler radar data," Journal of the Atmospheric Sciences, vol. 48, pp. 876-890, 1991.

[10] "A simple adjoint method of wind analysis for single-Doppler data," Journal of Atmospheric \& Oceanic Technology, vol. 9, no. 5, pp. 588-598, 1992.

[11] C.-J. Qiu, A.-M. Shao, S. Liu, and Q. Xu, "A two-step variational method for three-dimensional wind retrieval from single Doppler radar," Meteorology and Atmospheric Physics, vol. 91, no. 1-4, pp. 1-8, 2006.

[12] P. W. Chan, "A significant wind shear event leading to aircraft diversion at the Hong Kong international airport," Meteorological Applications, vol. 19, no. 1, pp. 10-16, 2012.

[13] R. A. Pielke, W. R. Cotton, R. L. Walko et al., "A comprehensive meteorological modeling system-RAMS," Meteorology and Atmospheric Physics, vol. 49, no. 1-4, pp. 69-91, 1992.

[14] W. C. Skamarock and J. B. Klemp, "A time-split nonhydrostatic atmospheric model for weather research and forecasting applications," Journal of Computational Physics, vol. 227, no. 7, pp. 3465-3485, 2008.

[15] D. J. Carruthers, J. C. R. Hunt, and W. S. Weng, "A computational model of stratified turbulent airow over hills-owstar I," in Proceedings of the Envirosoft Computer Techniques in Environmental Studies, P. Zanetti, Ed., pp. 481-492, Springer, New York, NY, USA, 1988.

[16] J. G. Sela, "Spectral modeling at the national meteorological center," Monthly Weather Review, vol. 108, no. 9, pp. 1279-1292, 1980. 
[17] M. Farazmand and G. Haller, "Computing Lagrangian coherent structures from their variational theory," Chaos, vol. 22, no. 1, Article ID 013128, 2012.

[18] D. J. Carruthers, A. Ellis, J. C. R. Hunt, and P. W. Chan, "Modelling of wind shear downwind of mountain ridges at Hong Kong international airport," Meteorological Applications, vol. 21, no. 1, pp. 94-104, 2014.

[19] P. Tallapragada, S. D. Ross, and D. G. Schmale, "Lagrangian coherent structures are associated with fluctuations in airborne microbial populations," Chaos, vol. 21, no. 3, Article ID 033122, 2011.

[20] R. C. Gonzalez and R. E. Woods, Digital Image Processing, Prentice Hall, 2007.

[21] A. E. BozorgMagham, S. D. Ross, and I. Schmale, "Real-time prediction of atmospheric Lagrangian coherent structures based on forecast data: an application and error analysis," Physica D: Nonlinear Phenomena, vol. 258, pp. 47-60, 2013. 

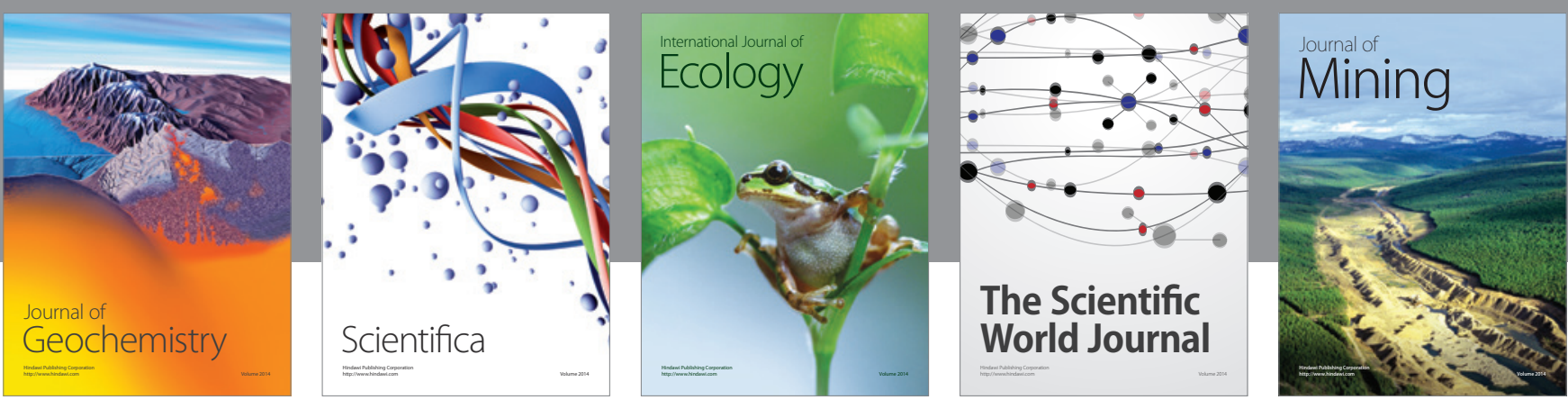

The Scientific World Journal
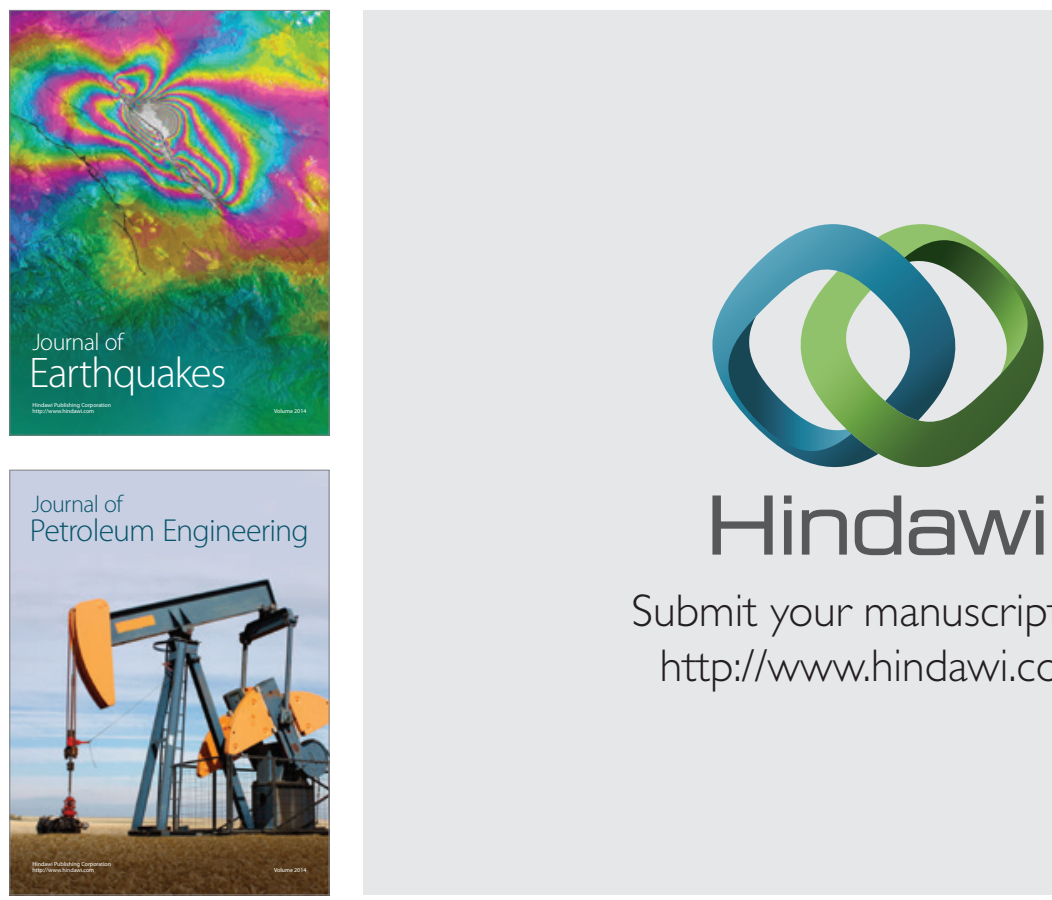

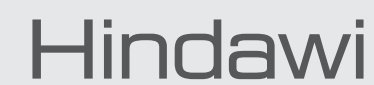

Submit your manuscripts at

http://www.hindawi.com
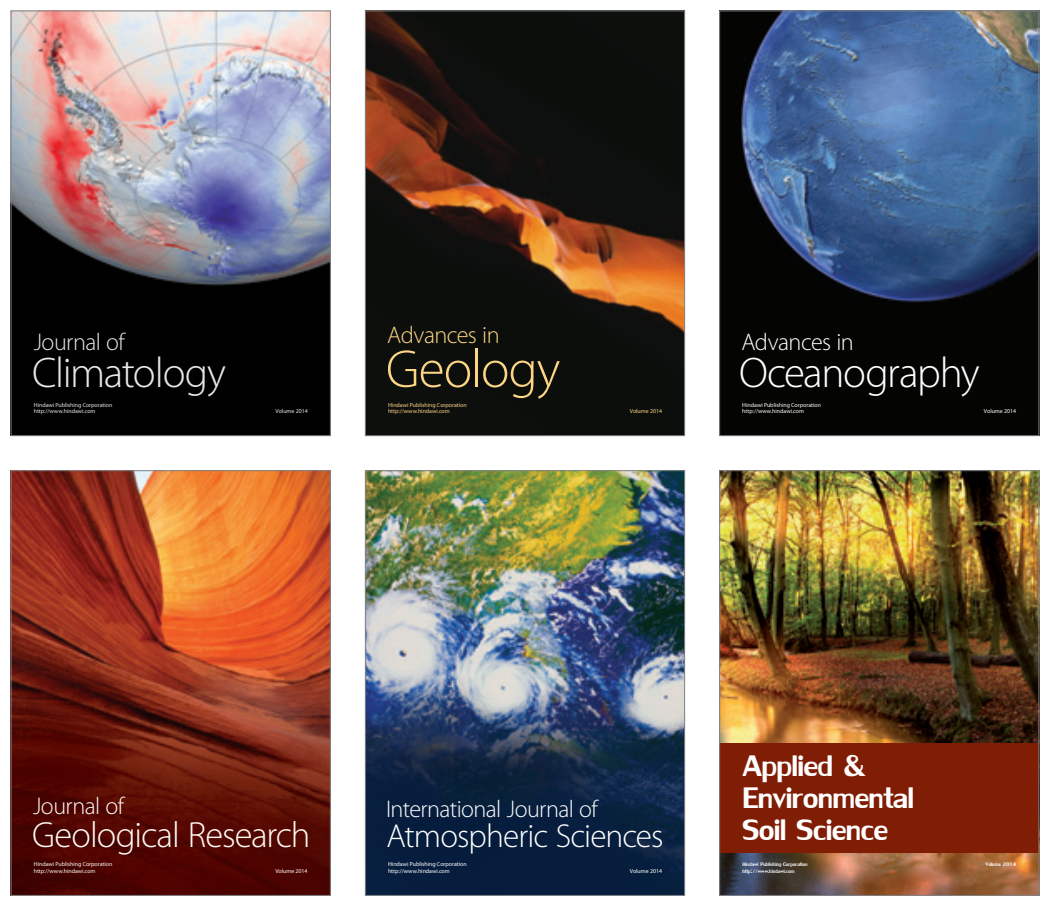
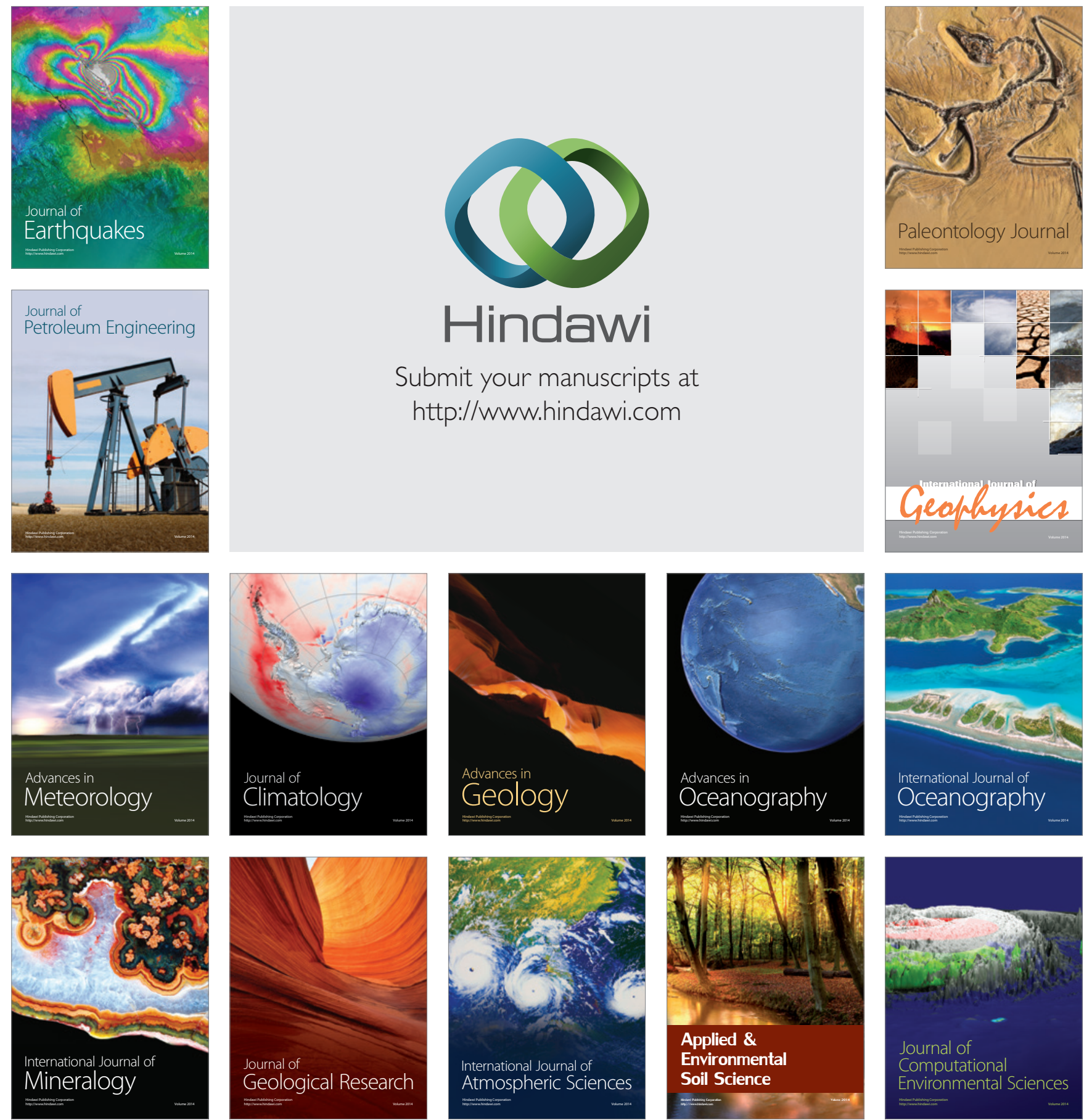